\title{
Physiological variation of liver iron concentration may not be dominantly responsible for the liver T1rho variations associated with age and gender
}

\author{
Yì Xiáng J. Wáng^ \\ Department of Imaging and Interventional Radiology, Faculty of Medicine, The Chinese University of Hong Kong, Shatin, Hong Kong SAR, China \\ Correspondence to: Dr. Yì Xiáng J. Wáng. Department of Imaging and Interventional Radiology, Faculty of Medicine, The Chinese University of \\ Hong Kong, Shatin, Hong Kong SAR, China. Email: yixiang_wang@cuhk.edu.hk.
}

Submitted Nov 05, 2020. Accepted for publication Nov 16, 2020.

doi: $10.21037 /$ qims-20-1250

View this article at: http://dx.doi.org/10.21037/qims-20-1250

Liver fibrosis, a common feature of almost all chronic liver diseases, involves the accumulation of collagen, proteoglycans, and other macromolecules in the extracellular matrix (ECM). T1rho (T1 $\rho)$ relaxation time describes spin-lattice relaxation in the rotation frame at the presence of an external radiofrequency pulse in the transverse plane. It has been demonstrated that T1rho elongation is a sensitive biomarker for collagen deposition, both in the liver and in the kidneys (1-3). Allkemper et al. (4) reported that T1rho value was significantly associated with the Child-Pugh staging of the patients. In patients with chronic liver diseases, Takayama et al. (5) demonstrated liver T1rho values showed significant positive correlations with the serum levels of total bilirubin, direct bilirubin, and indocyanine green (ICG-R15), and significant negative correlations with the serum levels of albumin and $\gamma$-glutamyl transpeptidase.

To translate liver T1rho MRI into clinical practise, it is important to understand physiological liver T1rho measure in healthy subjects. We have performed a healthy volunteer study in 62 women (mean age: 38.9 years; range, 18-75 years) and 34 men (mean age: 44.7 years; range, 24-80 years) (6). We reported that the female liver T1rho value ranged between 35.07 and $51.97 \mathrm{~ms}$ and showed an age-dependent decrease, with younger women having a higher measurement. The male liver T1rho value ranged between 34.94 and $43.39 \mathrm{~ms}$, with no evidential age dependence (Figure 1). Note the T1rho data acquisition had very high scan-rescan reproducibility (6-9). We have hypothesized that the observed age- and gender- related liver T1rho differences among healthy subjects might be largely due to the differences of liver iron deposition between men $v s$. women and between young subjects $v s$. older subjects (9). However, with re-consideration of the existing and also newly available data, we suggest that the observed age- and gender- related liver T1rho physiological differences are unlikely being primarily caused by liver iron concentration variation.

In healthy subjects there are iron storage depots containing ferritin and hemosiderin, which are located for the most part in the liver, spleen, and bone marrow. One-third of the body storage iron is found in the liver and its contents reflects the level of total body storage. If a subject is in iron balance, there is usually relatively little exchange between the stores and the rest of iron in the body. States of negative iron balance occur when the body requirements for iron exceed the amounts being absorbed from the gastrointestinal tract. This may be the result of physiological events such as growth spurts, menstruation and pregnancy, or of any pathological state that causes blood loss. There are numerous reports on the association between age and liver iron deposit. While variable results have been reported (the results partially affected by the study subjects' dietary style), there are sufficient evidences

^ ORCID: 0000-0001-5697-0717. 


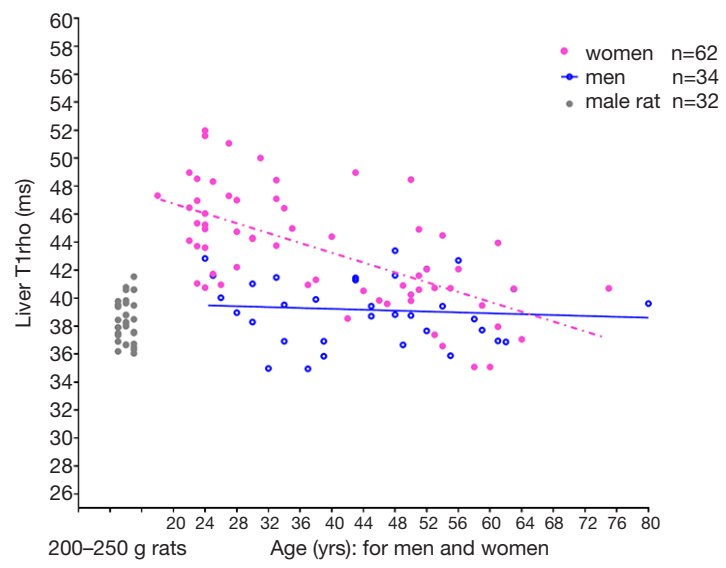

Figure 1 The relationship between age and liver T1rho. Women's liver T1rho shows an age-related decrease, with younger women showing a higher measurement. The men's liver T1rho has no evidential age dependence. After the age of 50 years, men and women have similar liver T1rho values. 32 young male rats have liver T1rho measures similar to those of men of various ages. Reproduced with permission from reference (6) and reference (7).

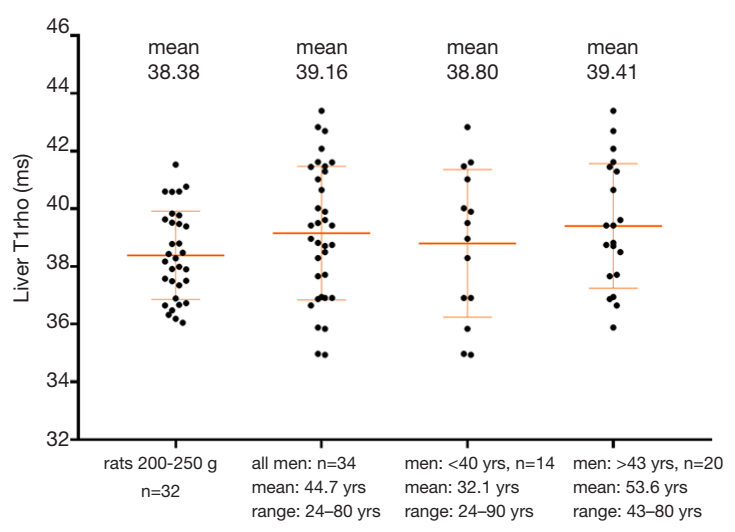

Figure 2 The relationship between age and liver T1rho in young male rats, total men participants, men $<40$ years old, and men $>43$ years old. Reproduced with permission from reference (6) and reference (7).

that, in healthy subjects without diet iron deficiency, liver iron concentration rises sharply in men toward the end of the adolescent growth spurt in the late teens and reached maximum before 40 years old. After 40 years old, liver iron level remains constant or slightly increases until approximately age 70 years, after which it may declines (10-13). This liver iron level physiological change pattern differs from the liver T1rho change over age in healthy men. Figure 1 and Figure 2 show there is no apparent aged related change observed for men of $24-80$ years. If higher liver iron in older subjects ( $>40$ years) caused T1rho shortening (as compared with younger subjects), then we would expect to see the $>43$ years group had shorter mean T1rho value than the $<40$ years group, which was not the case in Figure 2. Moreover, for all the male study subjects (mean: 44.7 years), the mean liver T1rho was similar to the young male rats of 200-250 gram.

On the other hand, age related liver iron increase has been observed with a number of MRI methods. Schwenzer et al. (14) reported that a statistically significant correlation of $\mathrm{T} 2$ * decrease over age in men (for men, Pearson correlation coefficients $\mathrm{r}=-0.30, \mathrm{P}<0.05, \mathrm{n}=44$; for women, $\mathrm{r}=-0.46, \mathrm{P}<0.0001, \mathrm{n}=85)$. In 34 men and 52 women, Metens et al. (15) investigated how normal liver parenchyma visibility on diffusion-weighted images (DWI) and apparent diffusion coefficient (ADC) quantification are influenced by age and iron content. Liver visibility (lower visibility denotes higher liver iron concentration) was better in young women but degrades with age in both genders. For $b=1,000 \mathrm{~s} / \mathrm{mm}^{2}$ images (other $b$-value images showed the same trend), inverse correlation between liver visibility score and age was significant with a Spearman coefficient $\mathrm{R}=-0.58$ in women $(\mathrm{P}<0.0001)$ and $\mathrm{R}=-0.44$ in men $(\mathrm{P}<0.01)$. The liver average ADC was negatively and weakly correlated with age (Spearman coefficient $\mathrm{R}=-0.23 ; \mathrm{P}=0.05)$, and the values were higher in women $\left(785 \pm 147 \times 10^{-6} \mathrm{~mm}^{2} / \mathrm{s}\right)$ than in men $\left(742 \pm 103 \times 10^{-6} \mathrm{~mm}^{2} / \mathrm{s}\right.$; $\mathrm{P}=0.027)$. Higher liver iron content is known to be associated with lower liver ADC measure (16).

In women, it is well known that liver iron concentration remains relatively low until after the fourth decade of life, after which they exhibit a steep rise. Maximum levels observed in women after menopause are approximately two thirds of those for men of comparable age (10-13,17-19). The relative low mean value among women in the 21 - to 40 -year-old group is associated with a high iron demand of menstruation during the childbearing age.

Recently, we performed a study with 30 men and 36 women on the correlation between intravoxel incoherent motion (IVIM) parameters and age. We demonstrated a trend of $D_{\text {slow }}$ decreasing related to healthy aging (20). Figure 3 shows a comparison of the relationship between $\mathrm{D}_{\text {slow }} v s$. age, and relationship between liver T1rho $v s$. age in men. A trend is noted for healthy aging associated with lower $\mathrm{D}_{\text {slow }}$, however, this trend does not exist for T1rho vs. aging, suggesting T1rho measure is at least less sensitive to liver iron concentration compared with $\mathrm{D}_{\text {slow }}$ measure. 

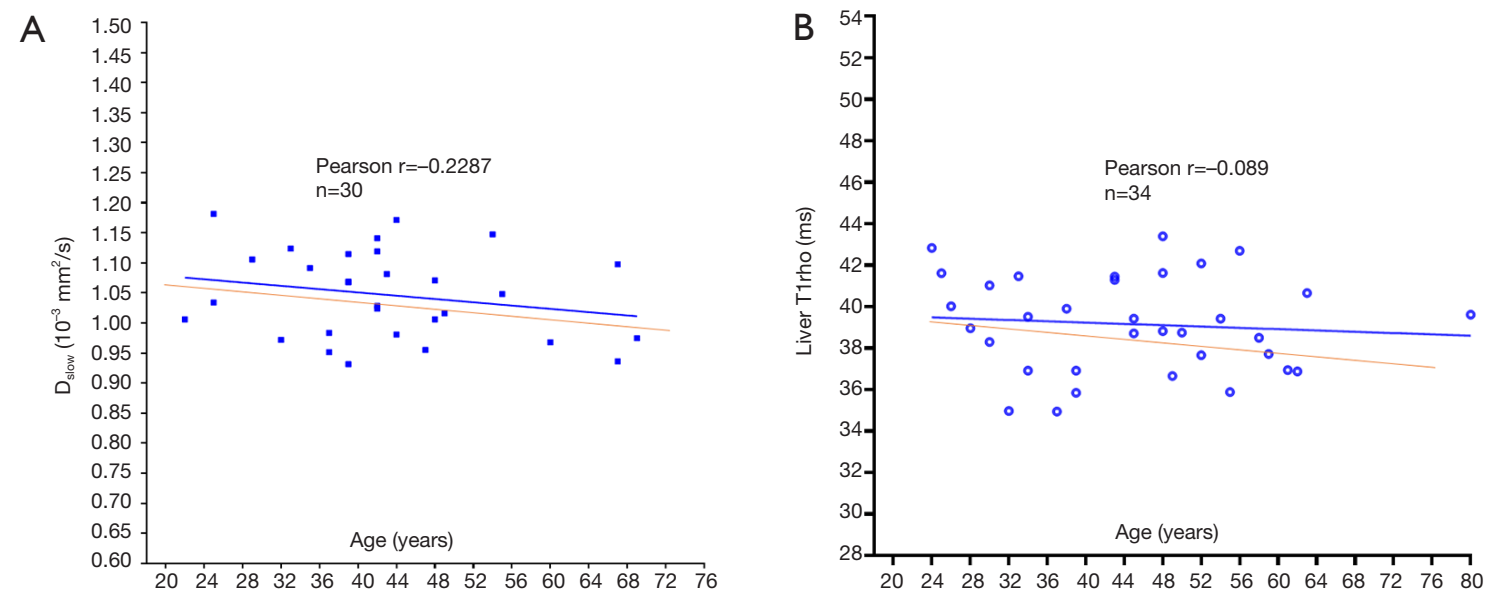

Figure 3 The relationship between $\mathrm{D}_{\text {slow }}$ of intravoxel incoherent motion analysis vs. age (study subjects: residents in Shenzhen, China) and liver T1rho vs. age in men (study subjects: residents in Hong Kong SAR, China). All study subjects were from South China thus with comparable dietary styles (the same for Figure 4). The men's liver T1rho has no evidential age dependence. The men's liver $\mathrm{D}_{\text {slow }}$ has a weak correlation with age (expected to be statistically significant if the sample size is increased to $\mathrm{n}=60$ ). Orange line presents the slope of $\mathrm{D}_{\text {slow }}$ decreasing over aging. Reproduced with permission from reference (6) and reference (20).
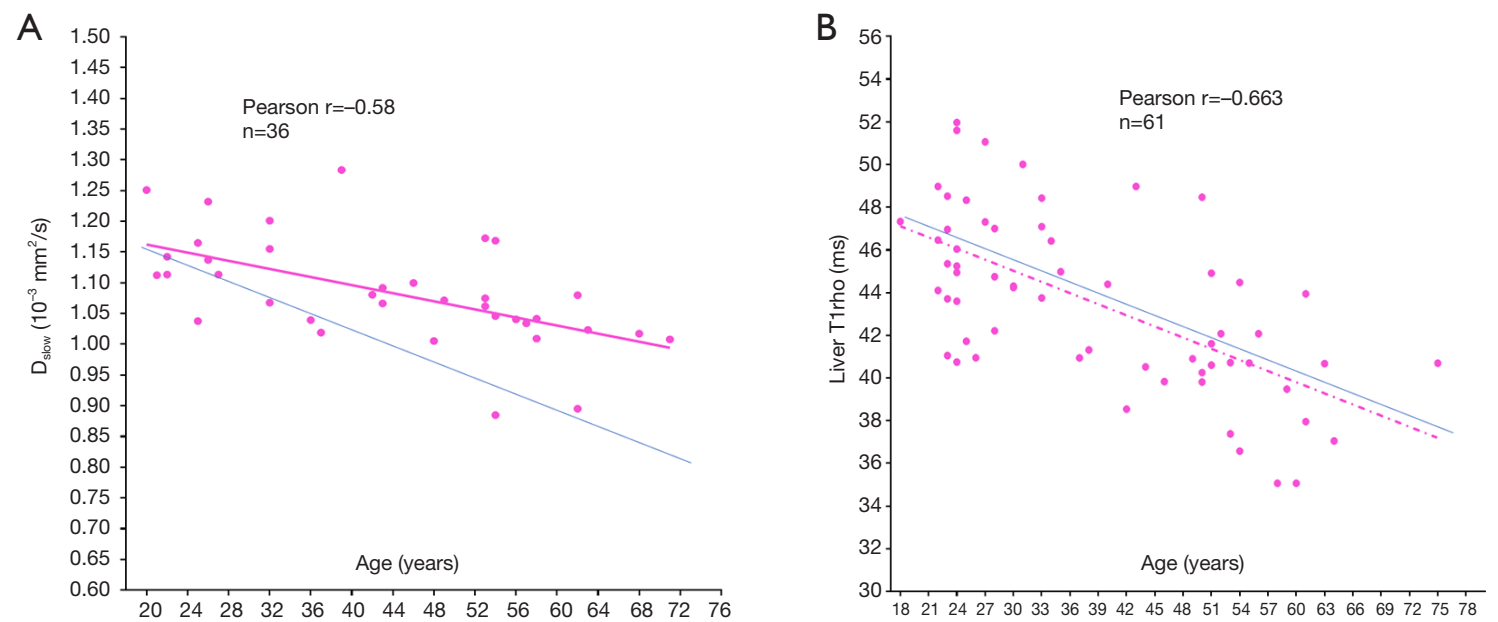

Figure 4 The relationship between liver $\mathrm{D}_{\text {slow }}$ of intravoxel incoherent motion analysis vs. age (A, study subjects: residents in Shenzhen, China) and liver T1rho vs. age in women (B, study subjects: residents in Hong Kong SAR, China). Age has a correlation of moderate strength both for $\mathrm{D}_{\text {slow }}(\mathrm{P}<0.001)$ and T1rho $(\mathrm{P}<0.0001)$. Blue line presents the slope of liver T1rho decreasing over aging, which is much steeper than that of $\mathrm{D}_{\text {slow }}$. Reproduced with permission from reference (6) and reference (20).

Figure 4 shows a comparison of the correlation between $\mathrm{D}_{\text {slow }} v s$. age and correlation between liver T1rho vs. age in women. Apparent and statistically significant inverse trends of healthy aging associated with both lower $\mathrm{D}_{\text {slow }}$ and shorter T1rho are noted. However, the opposite to men's results, the decreasing slope for liver T1rho over aging is steeper than that of $\mathrm{D}_{\text {slow }}$, suggesting the decreasing slope for liver T1rho over aging is not primarily caused by increased liver iron concentration over aging. If liver T1rho is not as sensitive to iron as $\mathrm{D}_{\text {slow }}$ to iron (as shown in men's results) but liver T1rho change still reflect liver iron change in women, then we would expect the women' liver T1rho slope over aging would be less steep than the slope of $\mathrm{D}_{\text {slow }}$ over aging. Secondly, between the age of 50-60 years liver iron concentration is expected to still substantially higher in men than in women; while for liver T1rho, men's and 
women's liver T1rho measures converged. Thus, we argue that the physiological T1rho change over aging is not dominantly caused by liver iron concentration variation. Compared with other MRI techniques such as $\mathrm{T} 2 *$ and diffusion imaging, T1rho is relatively insensitive to liver iron deposition at least when the iron concentration is within or close to physiological ranges. In a limited analysis of 16 liver cirrhosis patients with 4 of them had iron overload, Allkemper et al. (4) also did not find a correlation between liver T1rho and semi-quantitative liver iron overload.

Moreover, the opposite to our previous discussion (2), since young male rats had very similar liver T1rho to healthy men of various age $(6,7)$, it is more likely the liver fat content difference had not substantially contributed to the gender- and age- difference of T1rho demonstrated in our last health volunteer study. For subjects without apparent liver steatosis (i.e., $<5 \%$ fat content) and when fat suppression technique is applied for T1rho acquisition, the contributed from liver fat to T1rho shortening would be limited relative to the physiological variation of liver T1rho. Our previous analysis suggests that $10 \%$ additional liver fat contribute to $1.55 \mathrm{~ms}$ liver T1rho shortening (21). Also, in our previous report we noted that, for both women and men, no association was seen between body mass index and liver T1rho (6).

Our rat study demonstrated that the liver T1rho individual variation is unlikely due to measurement imprecision $(7,9)$. Instead, the variation more likely reflect genuine biochemical difference among individual rats. Based on our recent study on collagen's contribution to T1rho elongation $(2,22)$, we tentatively suggest that among healthy human subjects there are sufficient age and gender related macromolecular biochemical differences which contribute to the liver T1rho variations observed in healthy human beings (6). The effects of growth, aging and gender on liver tissue composition has long been a topic of research (23-26). The exact mechanism for the age and gender associated liver T1rho difference remains to be further clarified.

Furthermore, the high sensitivity of T1rho to collagen shall allow the possibility to image tumor ECM. Many solid tumors express high levels of various ECM molecules like fibrillar collagens, fibronectin, elastin, and laminins. In some tumors, the ECM compromises up to $60 \%$ of the tumor mass. Like the other components of the tumor microenvironment, the ECM in solid tumors differs significantly from that in normal organs. Source of these ECM molecules can be the tumor cells themselves, but to an even larger degree cancer-associated fibroblasts. The ECM does not only influence malignancy and growth of the tumor but also its response toward therapy. The infiltration of fibroblasts/myofibroblasts and the subsequent accumulation of significant amounts of collagenous ECM is observed in many solid tumors. This process, called desmoplasia, is strongly linked to poor prognosis and resistance to systemic therapy (27). Our recent work (2) shall justify further research into the role of T1rho for tumor characterisation as well as therapeutic monitoring (28-32).

\section{Acknowledgments}

Funding: This study was supported by Hong Kong GRF grants (Project No. 14109218).

\section{Footnote}

Provenance and Peer Review: This article was commissioned by the editorial office, Quantitative Imaging in Medicine and Surgery. The article did not undergo external peer review.

Conflicts of Interest: The author has completed the ICMJE uniform disclosure form (available at http://dx.doi. org/10.21037/qims-20-1250). YXJW serves as an unpaid Editor-in-Chief of Quantitative Imaging in Medicine and Surgery.

Open Access Statement: This is an Open Access article distributed in accordance with the Creative Commons Attribution-NonCommercial-NoDerivs 4.0 International License (CC BY-NC-ND 4.0), which permits the noncommercial replication and distribution of the article with the strict proviso that no changes or edits are made and the original work is properly cited (including links to both the formal publication through the relevant DOI and the license). See: https://creativecommons.org/licenses/by-nc-nd/4.0/.

\section{References}

1. Wang YX, Yuan J, Chu ES, Go MY, Huang H, Ahuja AT, Sung JJ, Yu J. T1rho MR imaging is sensitive to evaluate liver fibrosis: an experimental study in a rat biliary duct ligation model. Radiology 2011;259:712-9.

2. Zhao F, Zhou N, Wang JL, Zhou H, Zou LQ, Zhong WX, He J, Zheng CJ, Yan SX, Wáng YX. Collagen deposition in the liver is strongly and positively associated T1rho 
elongation while fat deposition is associated with T1rho shortening: an experimental study of methionine and choline-deficient (MCD) diet rat model. Quant Imaging Med Surg 2020;10:2307-21.

3. Hectors SJ, Bane O, Kennedy P, El Salem F, Menon M, Segall M, Khaim R, Delaney V, Lewis S, Taouli B. T1rho mapping for assessment of renal allograft fibrosis. J Magn Reson Imaging 2019;50:1085-91.

4. Allkemper T, Sagmeister F, Cicinnati V, Beckebaum S, Kooijman H, Kanthak C, Stehling C, Heindel W. Evaluation of fibrotic liver disease with whole-liver T1 $\rho$ MR imaging: a feasibility study at $1.5 \mathrm{~T}$. Radiology 2014;271:408-15.

5. Takayama Y, Nishie A, Asayama Y, Ushijima Y, Okamoto D, Fujita N, Morita K, Shirabe K, Kotoh K, Kubo Y, Okuaki T, Honda H. T1 $\rho$ Relaxation of the liver: A potential biomarker of liver function. J Magn Reson Imaging 2015;42:188-95.

6. Wáng YXJ, Deng M, Lin J, Kwok AWL, Liu EKW, Chen W. Age- and Gender-Associated Liver Physiological T1rho Dynamics Demonstrated with a Clinically Applicable Single-Breathhold Acquisition. SLAS Technol 2018;23:179-87.

7. Koon CM, Zhang X, Chen W, Chu ES, San Lau CB, Wáng YX. Black blood T1rho MR imaging may diagnose early stage liver fibrosis: a proof-of-principle study with rat biliary duct ligation model. Quant Imaging Med Surg 2016;6:353-63.

8. Wáng YXJ, Deng M, Lo GG, Liang D, Yuan J, Chen W. Breath-hold black-blood T1rho mapping improves liver T1rho quantification in healthy volunteers. Acta Radiol 2018;59:257-265.

9. Wáng YXJ, Chen W, Deng M. How liver pathologies contribute to T1rho contrast require more careful studies. Quant Imaging Med Surg 2017;7:608-13.

10. Zacharski LR, Ornstein DL, Woloshin S, Schwartz LM. Association of age, sex, and race with body iron stores in adults: analysis of NHANES III data. Am Heart J 2000;140:98-104.

11. Charlton RW, Hawkins DM, Mavor WO, Bothwell TH. Hepatic storage iron concentrations in different population groups. Am J Clin Nutr 1970;23:358-70.

12. Gautier du Défaix H, Puente R, Vidal B, Pérez E, Vidal H. Liver storage iron in normal population of Cuba. Am J Clin Nutr. 1980;33:133-6.

13. Wang JL, Shaw NS. Iron status of the Taiwanese elderly: the prevalence of iron deficiency and elevated iron stores. Asia Pac J Clin Nutr 2005;14:278-84.
14. Schwenzer NF, Machann J, Haap MM, Martirosian P, Schraml C, Liebig G, Stefan N, Häring HU, Claussen CD, Fritsche A, Schick F. T2* relaxometry in liver, pancreas, and spleen in a healthy cohort of one hundred twenty-nine subjects-correlation with age, gender, and serum ferritin. Invest Radiol 2008;43:854-60.

15. Metens T, Ferraresi KF, Farchione A, Moreno C, Bali MA, Matos C. Normal hepatic parenchyma visibility and ADC quantification on diffusion-weighted MRI at $3 \mathrm{~T}$ : influence of age, gender, and iron content. Eur Radiol 2014;24:3123-33.

16. Bülow R, Mensel B, Meffert P, Hernando D, Evert M, Kühn JP. Diffusion-weighted magnetic resonance imaging for staging liver fibrosis is less reliable in the presence of fat and iron. Eur Radiol 2013;23:1281-7.

17. Sturgeon $\mathrm{P}$, Shoden A. Total liver storage iron in normal populations of the USA. Am J Clin Nutr 1971;24:469-74.

18. Celada A, Herreros V, De Castro S. Liver iron storage in Spanish aging population. Am J Clin Nutr 1980;33:2662-4.

19. Weinfeld A, Lundin P, Lundvall O. Significance for the diagnosis of iron overload of histochemical and chemical iron in the liver of control subjects. J Clin Pathol 1968;21:35-40.

20. Huang H, Zheng CJ, Wang LF, Che-Nordin N, Wáng YX. Age and gender dependence of liver diffusion parameters and the possibility that intravoxel incoherent motion modelling of perfusion component is constrained by diffusion component. NMR Biomed 2020;e4449.

21. Zhao F, Zhou N, Wang X, Wang JL, Zhong WX, Deng M, Zheng CJ, He J, Yan SX, Wang YX. T1rho shortening effect of fat in liver steatosis after fat suppression: approximate estimation in a methionine and choline-deficient (MCD) diet rat model. Quant Imaging Med Surg 2021;11:870-5.

22. Hectors SJ. Is MRI relaxometry parameter T1 $\rho$ specific to fibrosis or confounded by concomitant pathological features? Quant Imaging Med Surg 2020;10:2408-10.

23. Campbell RM, Koster litz HW. The effects of growth and sex on the composition of the liver cells of the rat. J Endocrinol. 1950;6:308-18.

24. Trivedi P, Cheeseman P, Portmann B, Hegarty J, Mowat AP. Variation in serum type III procollagen peptide with age in healthy subjects and its comparative value in the assessment of disease activity in children and adults with chronic active hepatitis. Eur J Clin Invest 1985;15:69-74.

25. Ballestri S, Nascimbeni F, Baldelli E, Marrazzo A, Romagnoli D, Lonardo A. NAFLD as a Sexual Dimorphic 
Disease: Role of Gender and Reproductive Status in the Development and Progression of Nonalcoholic Fatty Liver Disease and Inherent Cardiovascular Risk. Adv Ther 2017;34:1291-326.

26. Kehlet SN, Willumsen N, Armbrecht G, Dietzel R, Brix S, Henriksen K, Karsdal MA. Age-related collagen turnover of the interstitial matrix and basement membrane: Implications of age- and sex-dependent remodeling of the extracellular matrix. PLoS One 2018;13:e0194458.

27. Henke E, Nandigama R, Ergün S. Extracellular Matrix in the Tumor Microenvironment and Its Impact on Cancer Therapy. Front Mol Biosci 2020;6:160.

28. Li LZ, Zhou R, Xu HN, Moon L, Zhong T, Kim EJ, Qiao H, Reddy R, Leeper D, Chance B, Glickson JD. Quantitative magnetic resonance and optical imaging biomarkers of melanoma metastatic potential. Proc Natl Acad Sci U S A 2009;106:6608-13.

29. Cao M, Ding W, Han X, Suo S, Sun Y, Wang Y, Qu J, Zhang X, Zhou Y. Brain T1 $\rho$ mapping for grading and

Cite this article as: Wáng YXJ. Physiological variation of liver iron concentration may not be dominantly responsible for the liver T1rho variations associated with age and gender. Quant Imaging Med Surg 2021;11(4):1668-1673. doi: 10.21037/qims20-1250
IDH1 gene mutation detection of gliomas: a preliminary study. J Neurooncol 2019;141:245-52.

30. Zhou N, Chu C, Dou X, Li M, Liu S, Guo T, Zhu L, Liu B, Chen W, He J, Yan J, Zhou Z, Yang X. Early Changes of Irradiated Parotid Glands Evaluated by T1rho-Weighted Imaging: A Pilot Study. J Comput Assist Tomogr 2017;41:472-76.

31. Hectors SJ, Moonen RP, Strijkers GJ, Nicolay K. T1 $\rho$ mapping for the evaluation of high intensity focused ultrasound tumor treatment. Magn Reson Med 2015;73:1593-601.

32. Karsdal MA, Nielsen MJ, Sand JM, Henriksen K, Genovese F, Bay-Jensen AC, Smith V, Adamkewicz JI, Christiansen C, Leeming DJ. Extracellular matrix remodeling: the common denominator in connective tissue diseases. Possibilities for evaluation and current understanding of the matrix as more than a passive architecture, but a key player in tissue failure. Assay Drug Dev Technol 2013;11:70-92. 